\title{
Colon epithelial cell-specific Bmal1 deletion impairs bone formation in mice
}

Running Head: Effects of Bmall deletion in colon epithelial cells on bone

Frank C. Ko ${ }^{1,2}$, Sarah B. Jochum ${ }^{3}$, Brittany M. Wilson ${ }^{1}$, Amal Adra ${ }^{1}$, Nikhil Patel ${ }^{1}$, Sherry Wilber $^{3}$, Maliha Shaikh ${ }^{3,4}$, Christopher Forsyth ${ }^{3,4}$, Ali Keshavarzian ${ }^{3,4}$, Garth R. Swanson ${ }^{3,4}$, D. Rick Sumner ${ }^{1,2}$

${ }^{1}$ Department of Anatomy\& Cell Biology

${ }^{2}$ Department of Orthopedic Surgery

${ }^{3}$ Division of Digestive Diseases and Nutrition, Department of Internal Medicine

${ }^{4}$ Rush Center for Integrated Microbiome and Chronobiology Research

Rush University Medical Center

Chicago, IL, 60612

Author Contributions: Study design: FCK, CF, AK, GRS, DRS. Study conduct and data collection: FCK, SBJ, BMW, AA, NP, RM, SW, MS. Data analysis: FCK, DRS. Drafting and revising manuscript content: all authors. Approving final and submitted version of manuscript: all authors. DRS takes responsibility for the integrity of the data analysis.

Keywords: Circadian rhythm, Bmal1, gut-bone axis, bone remodeling

Correspondence:

D. Rick Sumner, Ph.D.

Professor

Chair, Department of Anatomy \& Cell Biology

Armour Academic Center

600 S. Paulina St.

Suite 507

Chicago, IL 60612

Phone: 312-942-5511

Fax: 312-942-5744

Email: rick_sumner@rush.edu

Funding Source: NIH R21 AR075130 (DRS), T32AR073157 (DRS), R24AA026801 (AK), K01AR077679 (FCK), and Pfizer Competitive Grant Program: Inflammatory Bowel Disease 2019 (GRS) 


\section{Abstract}

47 The circadian clock system regulates multiple metabolic processes, including bone metabolism.

48 Previous studies have demonstrated that both central and peripheral circadian signaling regulate

49 skeletal growth and homeostasis. Disruption in central circadian rhythms has been associated

50 with a decline in bone mineral density and the global and osteoblast-specific disruption of clock

51 genes in bone tissue leads to lower bone mass. Gut physiology is highly sensitive to circadian

52 disruption. Since the gut is also known to affect bone remodeling, we sought to test the

53 hypothesis that circadian signaling disruption in colon epithelial cells affects bone. We therefore

54 assessed structural, functional, and cellular properties of bone in 8 week old Ts4-Cre and Ts4-

$55 \mathrm{Cre} ; \mathrm{Bmal1}^{\mathrm{fl} / \mathrm{fl}}(\mathrm{cBmalKO})$ mice, where the clock gene Bmal1 is deleted in colon epithelial cells.

56 Axial and appendicular trabecular bone volume was significantly lower in cBmalKO compared

57 to Ts4-Cre 8-week old mice in a sex-dependent fashion, with male but not female mice showing

58 the phenotype. Similarly, the whole bone mechanical properties were deteriorated in cBmalKO

59 male mice. The tissue level mechanisms involved suppressed bone formation with normal

60 resorption, as evidenced by serum markers and dynamic histomorphometry. Our studies

61 demonstrate that colon epithelial cell-specific deletion of Bmal1 leads to trabecular and cortical

62 bone loss in male mice. 


\section{Introduction}

65 Circadian rhythms are endogenous 24-hour recurring behavioral, physiological, and metabolic

66 patterns that are orchestrated by the circadian clock $(1,2)$. Central circadian misalignment

67 occurs when there is a mismatch between an environmental cue (e.g., light) and the central clock

68 in the suprachiasmatic nucleus, while peripheral circadian misalignment occurs when there is a

69 mismatch between an environmental cue (e.g., time of feeding) and endogenous rhythms of a

70 specific organ (e.g., the gut). Disruption in circadian rhythms due to rotating-shift work or long

71 distance travel with jet leg, has been associated with several metabolic disorders, including

72 obesity, insulin resistance, hypertension, and diabetes (3-6). Clinical studies have also

73 demonstrated that skeletal deterioration is associated with disrupted circadian rhythms (7). For

74 example, in studies of sleep deprived individuals, circadian rhythm disruption is associated with

75 decreased bone mineral density, increased fracture risk, and lower serum bone formation markers

76 (8-12). The mechanisms of how the circadian clock system regulates bone metabolism and

77 health remain unclear (13).

79 Genes such as the brain and muscle ARNT-like protein-1 (Bmal1), circadian locomotor output

80 cycles kaput (Clock), Period1 (Per1), Period2 (Per2), cryptochrome1 (Cry1), and cryptochrome2

81 (Cry2) are integral to the circadian signaling pathway (14). These genes have also been shown

82 to regulate skeletal growth and bone remodeling. When Bmal1 was globally deleted in mice,

83 longitudinal skeletal growth and osteoblast and osteocyte numbers were decreased (15).

84 Osteoprogenitor and osteoblast-specific deletion of Bmal1 led to decreased bone due to increased

85 bone resorption (16). Global deletion of Per1/2 or Cry1/2, which are negative regulators of

86 circadian signaling, increased bone in mice (17). More recently, intestinal epithelium-specific 
87 deletion of Bmal1 was shown to decrease bone in mice (Villin-Cre;Bmall ${ }^{\mathrm{fl} / \mathrm{fl}}$ ) (18). With this

88 model, Bmal1 is deleted in both the small intestine and the large intestine (19) and likely in the

89 kidney as well since Villin-Cre is expressed in kidney proximal tubules $(20,21)$. The study

90 demonstrated that the bone deficit was driven by increased bone resorption due to vitamin D-

91 induced calcium malabsorption in the duodenum to maintain normocalcemia and suppressed

92 bone formation due to altered sympathetic tone. However, no study to date has examined the

93 effects of colon-specific Bmal1 deletion on bone.

95 We recently demonstrated that Bmall deletion in the colon epithelium does not cause ulcerative

96 colitis but still leads to mild colonic inflammation in mice [S]. Several studies show that

97 alterations in gut physiology, such as inflammatory bowel diseases, have consequences for

98 skeletal health (22-25) and moderate gut inflammation that does not cause weight loss leads to

99 bone loss in mice (26). We therefore sought to determine the effects of Bmal1 deletion in colon

100 epithelium on bone. We hypothesized that colon epithelial cell-specific deletion of Bmall will

101 decrease bone in mice.

102

103 Materials and Methods

104 Mouse

105 Animal studies were approved by the Rush University Medical Center Institutional Animal Care

106 and Use Committee. Ts4-Cre (Ts4-Cre) and Ts4-Cre;Bmal1 ${ }^{\mathrm{fl} / \mathrm{fl}}$ (cBmalKO) mice, generously

107 provided from the laboratory of Khashayarsha Khazaie $\mathrm{Ph} . \mathrm{D}$ (27), were bred in-house to

108 generate the experimental mice used in this study. We previously demonstrated that $\mathrm{cBmalKO}$

109 mice (experimental group) exhibit mild colon inflammation compared to Ts4-Cre mice (control 
110 group) [S]. At 3 weeks of age, male and female Ts4-Cre and cBmalKO mice were weaned and

111 group housed 2 to 5 mice per cage. Mice were maintained in a pathogen-free facility, subjected

112 to a 12/12 hour light/dark cycle, had ad libitum access to standard laboratory rodent chow and

113 water, and were sacrificed by $\mathrm{CO}_{2}$ inhalation followed by cardiac puncture at the age of 8 weeks

114 ( $\mathrm{n}=9-10 /$ genotype/sex). All tissues were harvested between 10 a.m. and 12 p.m.

Specimen harvesting and preparation

117 After 2 hours of fasting, body weight (Suppl. Table 1) was measured and blood samples were

118 collected by cardiac puncture. Serum was collected after centrifuging blood samples and stored

119 in $-80^{\circ} \mathrm{C}$ until analysis. Both femurs and L5 vertebra were cleaned of soft tissue and the right

120 femur and L5 vertebra were wrapped in saline-soaked gauze and stored at $-20^{\circ} \mathrm{C}$ and the left

121 femur was fixed and stored in $70 \%$ ethanol at room temperature.

123 Serum biochemistry

124 Serum levels of procollagen type $1 \mathrm{~N}$-terminal propeptide (P1NP, Rat/Mouse P1NP EIA, IDS,

125 Gaithersburg, MD) and collagen type 1 C-telopeptide (RatLaps CTX-1 EIA, IDS, Gaithersburg,

126 MD) were evaluated as per the manufacturer's instructions.

Microcomputed tomography

129 Micro-computed tomographic $(\mu \mathrm{CT})$ imaging was performed on the distal metaphysis and mid-

130 diaphysis of the right femur and the whole L5 vertebra using a high-resolution laboratory

131 imaging system ( $\mu$ CT50, Scanco Medical AG, Brüttisellen, Switzerland) in accordance with the

132 American Society of Bone and Mineral Research (ASBMR) guidelines for the use of $\mu \mathrm{CT}$ in 
133 rodents (28). Scans were acquired using a $7.4 \mu \mathrm{m}^{3}$ isotropic voxel, $70 \mathrm{kVp}$ and $114 \mu \mathrm{A}$ peak x-

134 ray tube potential and intensity, $300 \mathrm{~ms}$ integration time, and were subjected to Gaussian

135 filtration. The distal metaphyseal region for analysis of trabecular bone began $200 \mu \mathrm{m}$ ( 27 slices)

136 proximal to the distal growth plate and extended proximally $10 \%$ of the femur length, and the

137 trabecular compartment was segmented from the cortical bone by manual contouring. In L5

138 vertebra, cortical bone was separated from cancellous bone by manual contouring and the region

139 of interest included the region between the end plates. Cortical bone morphology was evaluated

140 in the femoral mid-diaphysis in a region that started at $55 \%$ of the bone length proximal to the

141 femoral head and extended $10 \%$ of the femur length distally. Thresholds of 350 and $460 \mathrm{mg}$

$142 \mathrm{HA} / \mathrm{cm}^{3}$ were used for evaluation of trabecular and cortical bone, respectively. Trabecular bone

143 outcomes included trabecular bone volume fraction (BV/TV, $\left.\mathrm{mm}^{3} / \mathrm{mm}^{3}\right)$, thickness $(\mathrm{Tb} . \mathrm{Th}, \mathrm{mm})$,

144 and separation (Tb.Sp, mm). Cortical bone outcomes included cortical tissue mineral density

145 (Ct.TMD, mg HA $/ \mathrm{cm}^{3}$ ), cortical thickness (Ct.Th, mm), total cross-sectional, cortical bone, and

146 medullary areas (TA, BA, and $\mathrm{MA}, \mathrm{mm}^{2}$ ), and the maximum and minimum moments of inertia

$147\left(\mathrm{I}_{\max }\right.$ and $\left.\mathrm{I}_{\min }, \mathrm{mm}^{4}\right)$.

148

149 Mechanical testing

150 The right frozen femurs were thawed and subjected to three-point bending by a materials testing

151 machine (Criterion 43, MTS Systems, Eden Prairie, MN) (29). To determine the stiffness

$152(\mathrm{~N} / \mathrm{mm})$ and $\max$ load $(\mathrm{N})$, the femur was loaded to failure on the anterior surface at a constant

153 displacement rate of $0.03 \mathrm{~mm} / \mathrm{sec}$ with the two lower support points spaced $8 \mathrm{~mm}$ apart (30).

154 Force-displacement data were acquired at $30 \mathrm{~Hz}$. The frozen L5 vertebrae were thawed and

155 subjected to compression testing at a constant displacement rate of $0.02 \mathrm{~mm} / \mathrm{sec}$. The bottom 
156 endplate was fixed by cyanoacrylate glue. Force-displacement data were acquired at $30 \mathrm{~Hz}$ and

157 stiffness and max load were calculated. Based on the $\mu \mathrm{CT}$ and mechanical testing data, we

158 estimated the cortical bone elastic modulus following methods previously published (29).

160 Static and dynamic histomorphometry

161 Static and dynamic histomorphometric analyses were performed according to the criteria

162 established by the ASBMR (31). Calcein was administered at 2 and 7 days prior to sacrifice.

163 Femurs were dehydrated and embedded in poly methyl methacrylate. $5 \mu \mathrm{m}$ thick coronal

164 sections were stained with Goldner's Trichrome for evaluation of static histomorphometric

165 parameters (osteoblast surface/bone surface and osteoclast surface/bone surface) (32) or left

166 unstained for evaluation of the fluorochrome labels. Mineralizing surface per bone surface

167 (MS/BS, \%) and mineral apposition rate (MAR, $\mu \mathrm{m} /$ day) were measured on unstained sections to

168 calculate bone formation rate (BFR, $\left.\mu \mathrm{m}^{3} / \mu \mathrm{m}^{2} / \mathrm{day}\right)$. All measurements were performed using an

169 Osteomeasure image analyzer.

$171 \quad$ Statistical analysis

172 All data were checked for normality, and standard descriptive statistics were calculated. Two-

173 way ANOVA was used to test the main effects of Bmall deletion (gene) and sex and their

174 interaction on outcome parameters. Tukey's HSD post hoc comparisons of means test was used

175 to identify significant differences between groups. Differences were considered significant at $p$

$176<0.05$. Data are reported as mean \pm SD. 
Distal femoral metaphyseal trabecular bone mass was reduced by $35 \%$ due to Bmal1 deletion in male mice (Figure 1A). This was driven by thinning of trabeculae (-16\%) and increased trabecular separation $(+13 \%)$. L5 vertebral trabecular bone mass was decreased by $21 \%$ in male mice due to Bmall deletion (Figure 1B). This was primarily driven by thinning of trabeculae by $14 \%$ as trabecular separation remained similar. Deletion of Bmal1 in female mice did not lead to alterations in trabecular bone properties of the distal metaphyseal femur or L5 vertebra.

(Table 2). Similarly, total area (-12\%), bone area (-16\%), maximum moment of inertia (-25\%),

Cre male mice while medullary area and cortical bone tissue mineral density remained the same.

In female mice, Bmal1 deletion led to higher cortical bone tissue mineral density $(+7.5 \%)$.

191 Figure 1. Microarchitecture properties of distal metaphyseal femur (A) and L5 vertebra (B). $192 \mu \mathrm{CT}$ images from male Ts4-Cre and cBmalKO mice. Fem = femoral; L5 = L5 vertebral; BV/TV $193=$ bone volume/total volume; $\mathrm{Tb} . \mathrm{Th}=$ trabecular thickness; Tb.Sp = trabecular separation. Data 194 presented as Mean \pm SD

A

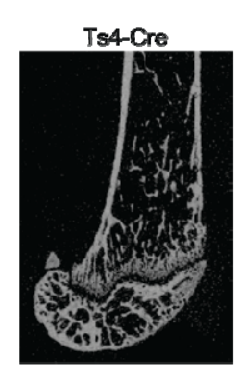

B

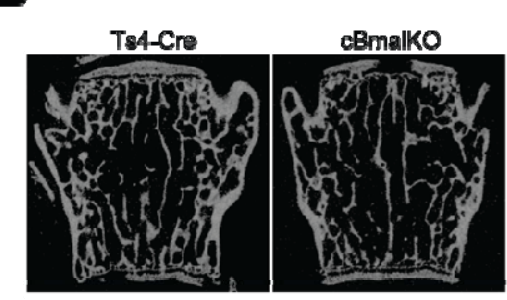

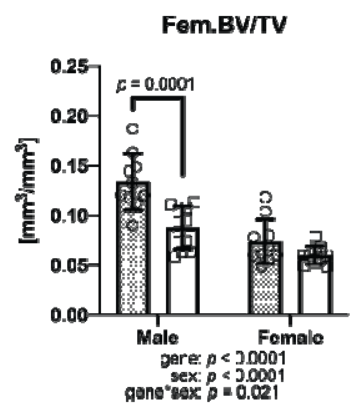

LE.BVTV

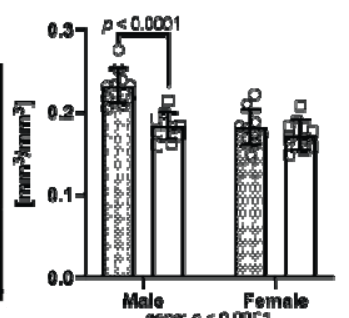

ecrep poponc

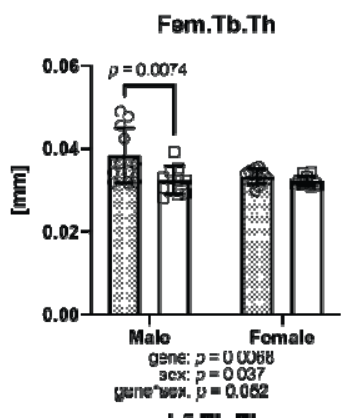

L6.Tb.Th

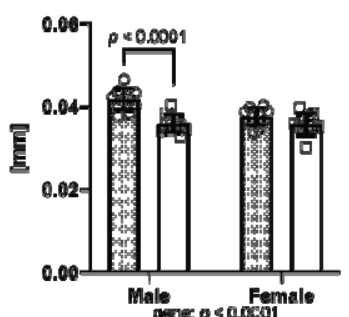

orex $p$ e $00 x 91$
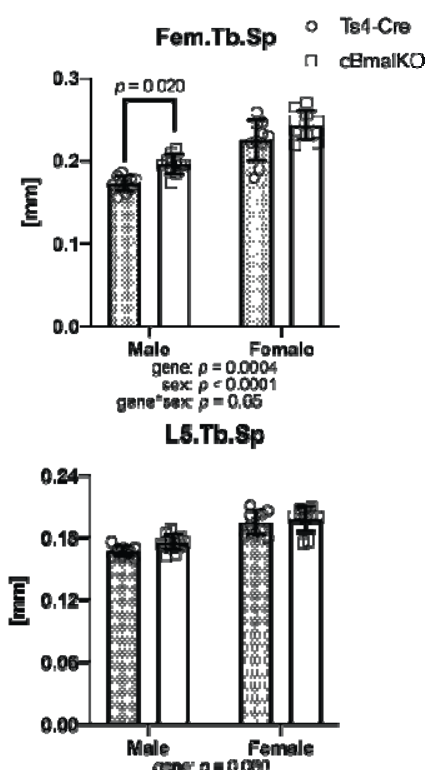

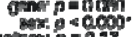

Table 2. Diaphyseal femoral cortical bone parameters. Ct.Th $=$ cortical thickness; TA $=$ total 196 
197 density; $\mathrm{I}_{\max }=$ maximum moment of inertia; $\mathrm{I}_{\min }=$ minimum moment of inertia. Data presented 198 as Mean $\pm \mathrm{SD} ; * * * p<0.0005 ; * * p<0.005 ; * p<0.05 ; v s$. Ts4-Cre within the same sex

\begin{tabular}{l|cc|cc|ccc}
\hline \hline & \multicolumn{2}{|c|}{ Male } & \multicolumn{2}{c|}{ Female } & \multicolumn{3}{c}{$p$ value } \\
& Ts4-Cre & cBmalKO & Ts4-Cre & cBmalKO & gene & sex & gene*sex \\
\hline Ct.Th $[\mathbf{m m}]$ & $0.16 \pm 0.02$ & $0.14 \pm 0.01^{*}$ & $0.15 \pm 0.004$ & $0.14 \pm 0.01$ & 0.004 & 0.013 & 0.056 \\
TA $\left[\mathbf{m m}^{2}\right]$ & $1.92 \pm 0.17$ & $1.69 \pm 0.12^{* *}$ & $1.64 \pm 0.12$ & $1.57 \pm 0.11$ & 0.0011 & $<0.0001$ & 0.073 \\
MA $\left[\mathbf{m m}^{2}\right]$ & $1.18 \pm 0.10$ & $1.07 \pm 0.08$ & $1.04 \pm 0.09$ & $0.99 \pm 0.08$ & 0.011 & 0.0004 & 0.30 \\
BA $\left[\mathbf{m m}^{2}\right]$ & $0.74 \pm 0.10$ & $0.62 \pm 0.05^{* * *}$ & $0.61 \pm 0.04$ & $0.58 \pm 0.05$ & 0.0005 & $<0.0001$ & 0.019 \\
Ct.TMD $\left[\mathbf{m g ~ H A} / \mathbf{c m}^{3}\right]$ & $971 \pm 66$ & $984 \pm 64$ & $969 \pm 63$ & $1042 \pm 33^{*}$ & 0.024 & 0.14 & 0.11 \\
$\mathbf{I}_{\max }\left[\mathbf{m m}^{4}\right]$ & $0.24 \pm 0.05$ & $0.18 \pm 0.02^{* * *}$ & $0.17 \pm 0.02$ & $0.15 \pm 0.02$ & 0.0003 & $<0.0001$ & 0.027 \\
$\mathbf{I}_{\min }\left[\mathbf{m m}^{4}\right]$ & $0.14 \pm 0.03$ & $0.10 \pm 0.02^{* *}$ & $0.10 \pm 0.01$ & $0.10 \pm 0.01$ & 0.001 & 0.0002 & 0.021 \\
\hline \hline
\end{tabular}

199 Femoral diaphyseal stiffness (-23\%) and max load (-26\%) were lower in cBmalKO male mice

200 compared to Ts4-Cre male mice (Fig 2). While L5 vertebral stiffness was not altered by the

201 Bmall deletion, max load was lower by $26 \%$ in cBmalKO male mice. Similar to other skeletal

202 parameters, female cBmalKO mice exhibited comparable biomechanical properties to Ts4-Cre

203 female mice.

204 Figure 2. Biomechanical properties of femoral diaphysis and L5 vertebrae in Ts4-Cre and

205 cBmalKO mice. Fem $=$ femoral; L5 = L5 vertebral. Data presented as Mean \pm SD

Fem.Stiffiness
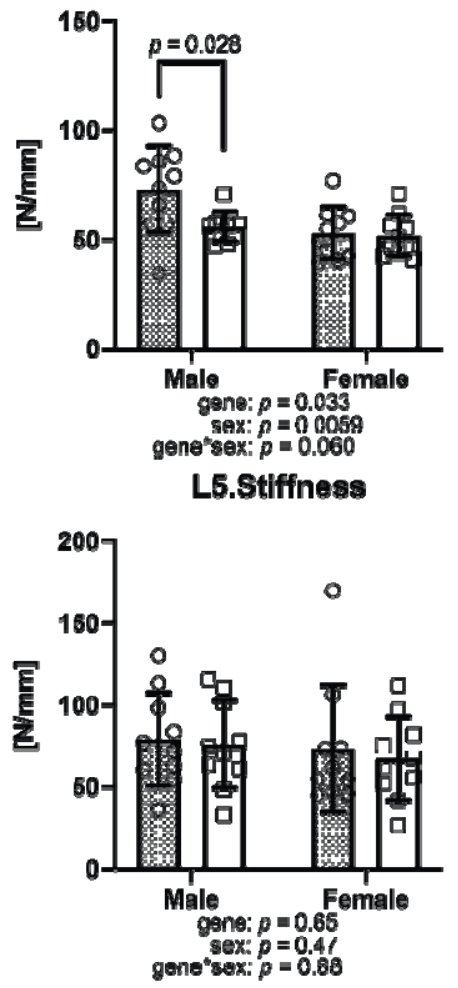

Fem.Max Load
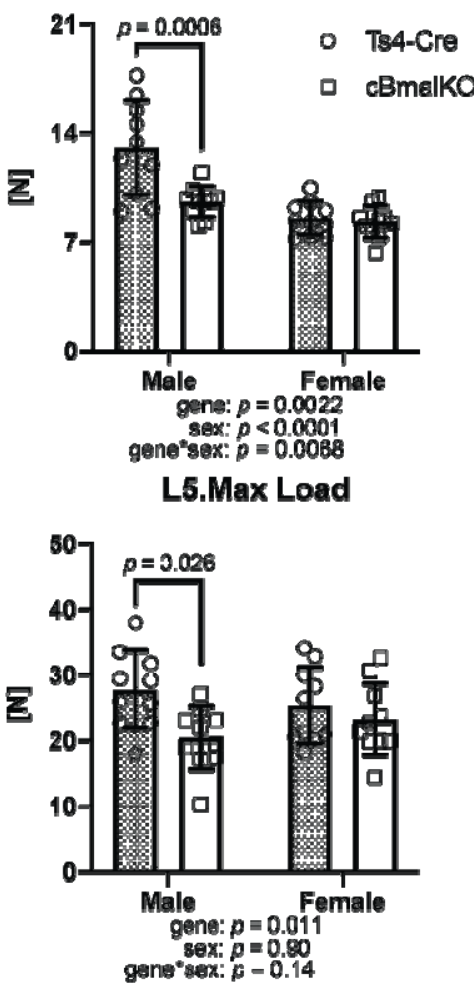
207 To determine if Bmal1 deletion alters bone resorption and formation, we assessed serum markers

208 of bone turnover by ELISA and static and dynamic histomorphometry of distal femoral

209 trabecular bone. Serum P1NP was 30\% lower in cBmalKO mice compared to Ts4-Cre mice in

210 the males, while no differences were observed between the two groups in female mice (Fig 3A).

211 Serum CTX-1 was unaffected by Bmal1 deletion or sex.

213 Static and dynamic histomorphometric parameters further confirmed that Bmal1 deletion impairs

214 bone formation (Fig 3B). In male cBmalKO mice, Ob.S/BS was lower by $42 \%$ compared to

215 Ts4-Cre mice whereas Oc.S/BS was similar. Bmal1 deletion in colon also led to 30\% lower

216 MAR and 65\% lower BFR/BS in male mice, but not in the females.

217 Figure 3. Serum bone remodeling markers (A) and static and dynamic histomorphometry (B).

218 Trichrome and fluorochrome images from male Ts4-Cre and cBmalKO mice. Ob.S/BS = 219 osteoblast surface/bone surface; Oc.S/BS = osteoclast surface/bone surface; MAR = mineral 220 apposition rate; $\mathrm{BFR} / \mathrm{BS}=$ bone formation rate/bone surface. Data presented as Mean $\pm \mathrm{SD}$

A

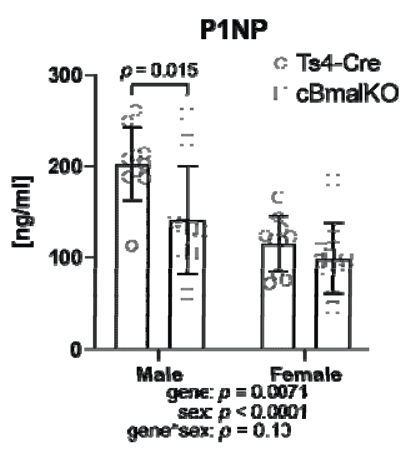

CTX-1

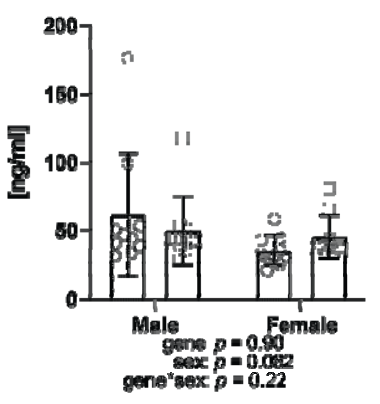

B
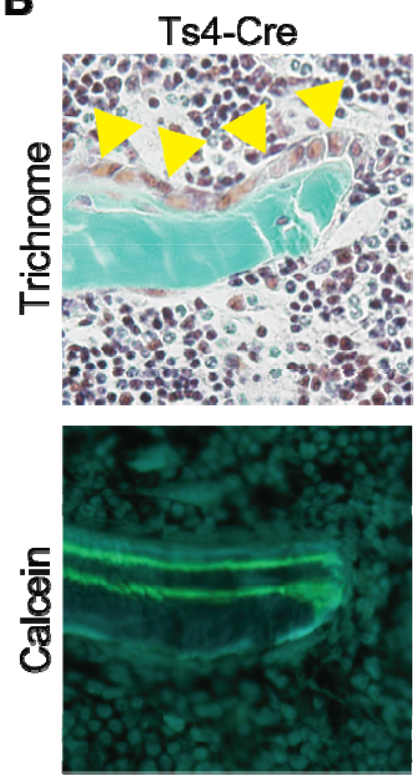

cBmalKO
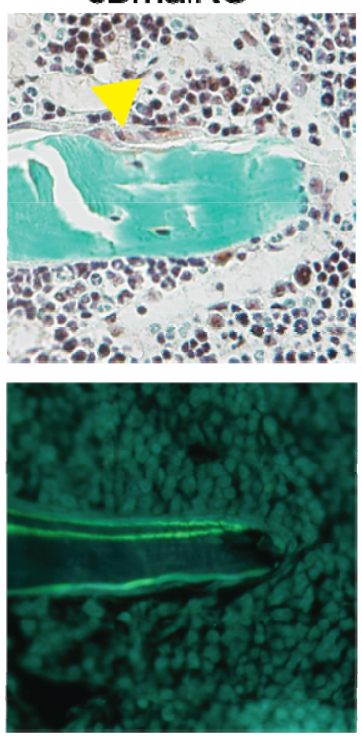

Ob.S/BS

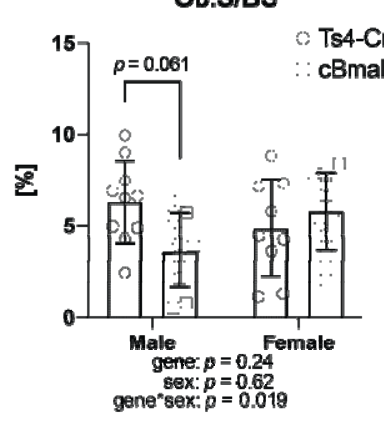

MAR

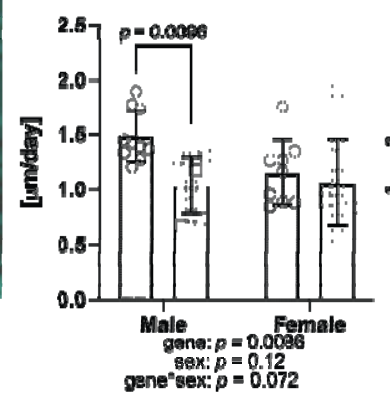

Oc.S/BS

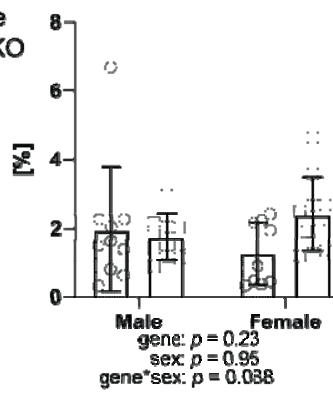

BFR/BS

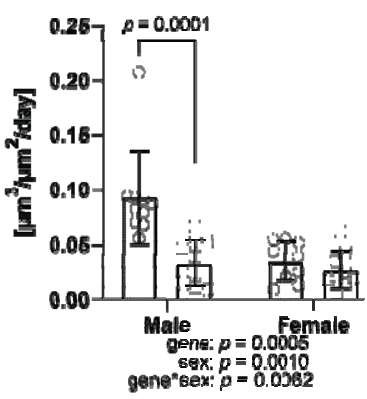




\section{Discussion}

223 This is the first study to demonstrated that colon epithelial cell-specific deletion of Bmal1 leads

224 to skeletal deterioration. The effect includes both cortical and trabecular bone with consequences

225 for biomechanical properties in the appendicular and axial skeleton, but only in male mice.

226 These differences appear to be driven by impaired bone formation in cBmalKO male mice, both

227 evidenced by both the serum bone formation marker P1NP and dynamic histomorphometry.

229 Our findings are consistent with a previous study where deletion of Bmall in cells expressing

230 Villin-Cre was associated with decreased bone (18), but the tissue level mechanism was not

231 consistent (no change in bone resorption vs. elevated resorption in the other study). The

232 differences may be attributed to the specificity of Bmall deletion, where Villin-Cre deletes

233 Bmal1 in both the small and large intestine and the kidney, whereas Ts4-Cre used in our studies

234 only deletes Bmal1 in the large intestine and the very distal ileum $(19,33)$. The age at which the

235 skeletal phenotyping was performed may also explain the differences observed (8 weeks old in

236 our study vs. 16 weeks old).

238 The age (8 weeks old) at which we assessed the skeleton of Ts4-Cre and cBmalKO mice

239 corresponds to the age when mice reach their peak trabecular bone mass in both the appendicular

240 and axial bone (34). In the femoral diaphysis, the cross-sectional area and cortical bone

241 thickness continue to increase beyond 8 weeks (35). Our findings suggest that in male mice, the

242 acquisition of peak trabecular bone mass is impaired by colon-specific Bmall deletion. The

243 architectural and functional deficits in the femoral diaphysis may continue to persist as the mice 
244 age, but further study is needed to confirm that. Whether female mice will continue to be

245 protected from skeletal deterioration as they age is unclear.

246

247 Our study surprisingly revealed that female mice were protected from structural and functional

248 skeletal deficits due to Bmall deletion in colon epithelial cells. Studies that examine whether the

249 gut-bone axis exhibits sexual dimorphism are limited, but a previous study demonstrated that

250 female mice were protected from increased gut permeability from antibiotics and subsequent

251 deteriorations in the skeleton (36). Also, ulcerative colitis patients showed decrease in bone

252 mineral density that was more significant in male than in female patients (37). Since studies

253 have suggested that estradiol has protective effects against metabolic disorders such as obesity,

254 osteoporosis, and diabetes (38-40), female mice in our study may similarly be protected from

255 colon-specific Bmal1 deletion-mediated skeletal deterioration due to higher levels of estradiol

$256(41,42)$.

257

258 We acknowledge the limitation of our studies where the underlying molecular mechanisms of

259 colon Bmal1 deletion-mediated skeletal deterioration were not examined, although we did

260 identify one of the tissue level mechanisms (suppressed bone formation). While the mild

261 inflammation observed in colon in this model [S] may suggest osteoclast-mediated bone

262 resorption as shown in other studies (43-45), our studies of serum bone remodeling markers and

263 dynamic histomorphometry suggest that Bmal1 deletion is likely inhibiting factors in the colon

264 that promote bone formation. Also, although we observed mild colon inflammation, cBmalKO

265 mice did not exhibit increased gut leakiness compared to Ts4-Cre mice [S], suggesting that other

266 gut-derived factors are likely regulating skeletal homeostasis. Gut microbiome and gut-derived 
267 hormones are known to regulate osteoblast function and thereby bone formation $(46,47)$. The

268 circadian rhythm of gut-derived plasma short chain fatty acids (SCFAs) has been shown to be

269 disrupted among shift workers (48), and along with a previous study that shows the importance

270 of SCFA butyrate to bone formation (49), the skeletal pathologies seen in our studies may be

271 attributed to the disruption of gut microbiota due to a disruption in circadian signaling in gut.

272

273 In conclusion, our study demonstrates that colon epithelial cell-specific deletion of Bmal1 leads

274 to trabecular and cortical bone loss in male mice, whereas female mice are unaffected. This

275 suggests that strategies that maintain the circadian rhythm in colon may prevent subsequent

276 skeletal deterioration observed in sleep-deprived individuals.

\section{Acknowledgments}

279 This study was supported by NIH R21AR075130 (DRS), T32AR073157 (DRS), R24AA026801

280 (AK), K01AR077679 (FCK), and Pfizer Competitive Grant Program: Inflammatory Bowel

281 Disease 2019 (GRS). The content is solely the responsibility of the authors and does not

282 necessarily represent the official views of the National Institutes of Health. AK would also like 283 to acknowledge philanthropy funding from The Johnson Family, Mrs. Barbara and Mr. Larry

284 Field, Mrs. Ellen and Mr. Philip Glass, Mrs. Marcia and Mr. Silas Keehn and the Sklar Family.

285 We thank Dr. Khashayarsha Khazaie for his scientific support for this project. Rush University

286 Medical Center MicroCT/Histology Core provided experimental support. 


\section{Reference:}

288 1. Potter GD, Skene DJ, Arendt J, Cade JE, Grant PJ, Hardie LJ. Circadian Rhythm and Sleep Disruption: Causes, Metabolic Consequences, and Countermeasures. Endocr Rev.

290 2016;37(6):584-608.

2912 2. Golombek DA, Rosenstein RE. Physiology of circadian entrainment. Physiol Rev. 292 2010;90(3):1063-102.

293 3. Stenvers DJ, Scheer F, Schrauwen P, la Fleur SE, Kalsbeek A. Circadian clocks and 294 insulin resistance. Nat Rev Endocrinol. 2019;15(2):75-89.

295 4. Lemmer B, Oster H. The Role of Circadian Rhythms in the Hypertension of Diabetes 296 Mellitus and the Metabolic Syndrome. Curr Hypertens Rep. 2018;20(5):43.

297 5. Maury E. Off the Clock: From Circadian Disruption to Metabolic Disease. Int J Mol Sci. 298 2019;20(7).

299 6. Orihara K, Haraguchi A, Shibata S. Crosstalk Among Circadian Rhythm, Obesity and 300 Allergy. Int J Mol Sci. 2020;21(5).

301 7. Specker BL, Binkley T, Vukovich M, Beare T. Volumetric bone mineral density and 302 bone size in sleep-deprived individuals. Osteoporos Int. 2007;18(1):93-9.

303 8. Quevedo I, Zuniga AM. Low bone mineral density in rotating-shift workers. J Clin 304 Densitom. 2010;13(4):467-9.

305 9. Kim BK, Choi YJ, Chung YS. Other than daytime working is associated with lower bone 306 mineral density: the Korea National Health and Nutrition Examination Survey 2009. Calcif 307 Tissue Int. 2013;93(6):495-501.

308 10. Feskanich D, Hankinson SE, Schernhammer ES. Nightshift work and fracture risk: the 309 Nurses' Health Study. Osteoporos Int. 2009;20(4):537-42.

310 11. Swanson CM, Shea SA, Wolfe P, Cain SW, Munch M, Vujovic N, Czeisler CA, Buxton 311 OM, Orwoll ES. Bone Turnover Markers After Sleep Restriction and Circadian Disruption: A Mechanism for Sleep-Related Bone Loss in Humans. J Clin Endocrinol Metab. 2017;102(10):3722-30.

12. Swanson CM, Kohrt WM, Wolfe P, Wright KP, Jr., Shea SA, Cain SW, Munch M, Vujovic N, Czeisler CA, Orwoll ES, Buxton OM. Rapid suppression of bone formation marker in response to sleep restriction and circadian disruption in men. Osteoporos Int. 2019;30(12):2485-93.

13. Swanson CM, Kohrt WM, Buxton OM, Everson CA, Wright KP, Jr., Orwoll ES, Shea SA. The importance of the circadian system \& sleep for bone health. Metabolism. 2018;84:28-43. 14. Cox KH, Takahashi JS. Circadian clock genes and the transcriptional architecture of the clock mechanism. J Mol Endocrinol. 2019;63(4):R93-R102.

15. Samsa WE, Vasanji A, Midura RJ, Kondratov RV. Deficiency of circadian clock protein BMAL1 in mice results in a low bone mass phenotype. Bone. 2016;84:194-203.

16. Takarada T, Xu C, Ochi H, Nakazato R, Yamada D, Nakamura S, Kodama A, Shimba S, Mieda M, Fukasawa K, Ozaki K, Iezaki T, Fujikawa K, Yoneda Y, Numano R, Hida A, Tei H, Takeda S, Hinoi E. Bone Resorption Is Regulated by Circadian Clock in Osteoblasts. J Bone Miner Res. 2017;32(4):872-81.

17. Fu L, Patel MS, Bradley A, Wagner EF, Karsenty G. The molecular clock mediates leptin-regulated bone formation. Cell. 2005;122(5):803-15.

330 18. Kawai M, Kinoshita S, Yamazaki M, Yamamoto K, Rosen CJ, Shimba S, Ozono K, 331

Michigami T. Intestinal clock system regulates skeletal homeostasis. JCI Insight. 2019;4(5). 
19. Madison BB, Dunbar L, Qiao XT, Braunstein K, Braunstein E, Gumucio DL. Cis elements of the villin gene control expression in restricted domains of the vertical (crypt) and horizontal (duodenum, cecum) axes of the intestine. J Biol Chem. 2002;277(36):33275-83. 20. Kucherlapati MH, Nguyen AA, Bronson RT, Kucherlapati RS. Inactivation of conditional $\mathrm{Rb}$ by Villin-Cre leads to aggressive tumors outside the gastrointestinal tract. Cancer Res. 2006;66(7):3576-83. Metzger D, Robine S. Tissue-specific and inducible Cre-mediated recombination in the gut epithelium. Genesis. 2004;39(3):186-93.

22. Zaiss MM, Jones RM, Schett G, Pacifici R. The gut-bone axis: how bacterial metabolites bridge the distance. J Clin Invest. 2019;130. the gut-bone axis - Physiological, pathophysiological and potential therapeutic implications. Peptides. 2020;125:170197.

24. Compston JE, Judd D, Crawley EO, Evans WD, Evans C, Church HA, Reid EM, Rhodes J. Osteoporosis in patients with inflammatory bowel disease. Gut. 1987;28(4):410. 25. Roux C, Abitbol V, Chaussade S, Kolta S, Guillemant S, Dougados M, Amor B, Couturier D. Bone loss in patients with inflammatory bowel disease: a prospective study. Osteoporos Int. 1995;5(3):156-60.

26. Irwin R, Raehtz S, Parameswaran N, McCabe LR. Intestinal inflammation without weight loss decreases bone density and growth. American journal of physiology Regulatory, integrative and comparative physiology. 2016;311(6):R1149-r57.

27. Khazaie K, Zadeh M, Khan MW, Bere P, Gounari F, Dennis K, Blatner NR, Owen JL, Klaenhammer TR, Mohamadzadeh M. Abating colon cancer polyposis by Lactobacillus acidophilus deficient in lipoteichoic acid. Proc Natl Acad Sci U S A. 2012;109(26):10462-7. 28. Bouxsein ML, Boyd SK, Christiansen BA, Guldberg RE, Jepsen KJ, Muller R. Guidelines for assessment of bone microstructure in rodents using micro-computed tomography. Journal of Bone and Mineral Research. 2010;25(7):1468-86.

29. Jepsen KJ, Silva MJ, Vashishth D, Guo XE, van der Meulen MC. Establishing biomechanical mechanisms in mouse models: practical guidelines for systematically evaluating phenotypic changes in the diaphyses of long bones. J Bone Miner Res. 2015;30(6):951-66.

30. Ko FC, Li J, Brooks DJ, Rutkove SB, Bouxsein ML. Structural and functional properties of bone are compromised in amyotrophic lateral sclerosis mice. Amyotroph Lateral Scler Frontotemporal Degener. 2018;19(5-6):457-62.

\section{Dempster DW, Compston JE, Drezner MK, Glorieux FH, Kanis JA, Malluche H,} Meunier PJ, Ott SM, Recker RR, Parfitt AM. Standardized nomenclature, symbols, and units for bone histomorphometry: A 2012 update of the report of the ASBMR Histomorphometry Nomenclature Committee. JBone MinerRes. 2013;28(1):2-17.

32. Ko FC, Martins JS, Reddy P, Bragdon B, Hussein AI, Gerstenfeld LC, Demay MB. Acute Phosphate Restriction Impairs Bone Formation and Increases Marrow Adipose Tissue in Growing Mice. J Bone Miner Res. 2016;31(12):2204-14.

\section{Saam JR, Gordon JI. Inducible gene knockouts in the small intestinal and colonic} epithelium. J Biol Chem. 1999;274(53):38071-82.

34. Glatt V, Canalis E, Stadmeyer L, Bouxsein ML. Age-related changes in trabecular architecture differ in female and male C57BL/6J mice. J Bone Miner Res. 2007;22(8):1197-207. 
35. Brodt MD, Ellis CB, Silva MJ. Growing C57B1/6 mice increase whole bone mechanical properties by increasing geometric and material properties. Journal of Bone and Mineral Research. 1999;14(12):2159-66. 36. Pusceddu MM, Stokes PJ, Wong A, Gareau MG, Genetos DC. Sexually Dimorphic Influence of Neonatal Antibiotics on Bone. J Orthop Res. 2019;37(10):2122-9. bone metabolism in inflammatory bowel disease: there is a difference between Crohn's disease and ulcerative colitis. Journal of internal medicine. 2000;247(1):63-70.

38. Brown LM, Clegg DJ. Central effects of estradiol in the regulation of food intake, body weight, and adiposity. J Steroid Biochem Mol Biol. 2010;122(1-3):65-73.

39. Christiansen C. Prevention and treatment of osteoporosis: a review of current modalities. Bone. 1992;13 Suppl 1:S35-9. 40. Liu S, Mauvais-Jarvis F. Minireview: Estrogenic protection of beta-cell failure in metabolic diseases. Endocrinology. 2010;151(3):859-64.

Estrogen weakens muscle endurance via estrogen receptor-p38 MAPK-mediated orosomucoid (ORM) suppression. Experimental \& molecular medicine. 2018;50(3):e463.

\section{Samuel P, Khan MA, Nag S, Inagami T, Hussain T. Angiotensin AT(2) receptor} contributes towards gender bias in weight gain. PLoS One. 2013;8(1):e48425.

43. Ciucci T, Ibanez L, Boucoiran A, Birgy-Barelli E, Pene J, Abou-Ezzi G, Arab N, Rouleau M, Hebuterne X, Yssel H, Blin-Wakkach C, Wakkach A. Bone marrow Th17 TNFalpha cells induce osteoclast differentiation, and link bone destruction to IBD. Gut. 2015;64(7):107281.

44. Ashcroft AJ, Cruickshank SM, Croucher PI, Perry MJ, Rollinson S, Lippitt JM, Child JA, Dunstan C, Felsburg PJ, Morgan GJ, Carding SR. Colonic dendritic cells, intestinal inflammation, and T cell-mediated bone destruction are modulated by recombinant osteoprotegerin. Immunity. 2003;19(6):849-61.

45. Ke K, Arra M, Abu-Amer Y. Mechanisms Underlying Bone Loss Associated with Gut Inflammation. Int J Mol Sci. 2019;20(24).

46. Behera J, Ison J, Tyagi SC, Tyagi N. The role of gut microbiota in bone homeostasis. Bone. 2020;135:115317.

408 47. Schiellerup SP, Skov-Jeppesen K, Windelov JA, Svane MS, Holst JJ, Hartmann B, Rosenkilde MM. Gut Hormones and Their Effect on Bone Metabolism. Potential Drug Therapies in Future Osteoporosis Treatment. Frontiers in endocrinology. 2019;10:75. 48. Swanson GR, Siskin J, Gorenz A, Shaikh M, Raeisi S, Fogg L, Forsyth C, Keshavarzian A. Disrupted diurnal oscillation of gut-derived Short chain fatty acids in shift workers drinking alcohol: Possible mechanism for loss of resiliency of intestinal barrier in disrupted circadian host. Transl Res. 2020;221:97-109.

49. Tyagi AM, Yu M, Darby TM, Vaccaro C, Li JY, Owens JA, Hsu E, Adams J, Weitzmann MN, Jones RM, Pacifici R. The Microbial Metabolite Butyrate Stimulates Bone Formation via T Regulatory Cell-Mediated Regulation of WNT10B Expression. Immunity. 2018;49(6):1116-31 e7. 